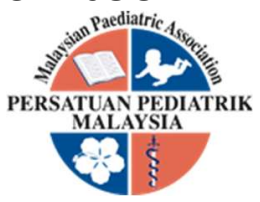

\title{
PASSIVE SMOKING AND CONGENITAL HEART DEFECTS IN OFFSPRING IN BANGLADESH
}

\author{
Mohammad Abdullah Al Mamun', Manzoor Hussain², Md. Khalid Ebna Shahid Khan ${ }^{3}$
}

\begin{abstract}
Objective: Maternal active smoking is significantly associated with an increased risk of congenital heart defects (CHDs), but the association between maternal passive smoking and risk of CHDs is inconclusive. This study was conducted to evaluate the effect of passive smoking and the risk of development of CHD among Bangladeshi population. Methodology: It was a case control study, conducted from July 2018 to July 2019 in Paediatric Cardiology department of Dhaka Shishu (Children) Hospital. Parents of the children with CHD visiting the out-patient department of Dhaka Shishu Hospital was considered as cases. Controls were taken from parents of the children who don't have congenital heart disease. Face-to-face interviews were conducted to collect information on maternal active and passive smoking. Data was collected by using a structured questionnaire containing all the variables of interest and analyzed by using SPSS version 21. Chi square test $\left(X^{2}\right)$, Odds ratio (OR) and 95\% confidence intervals $(\mathrm{Cls})$ were calculated to estimate the associations between maternal passive smoking and all CHDs. Risk factors on bivariate analysis were introduced into a logistic regression model as independent factors and dependent variable was CHDs to find out the association between CHDs and maternal passive smoking. Results: Among case group no mother was active smoker, $51.9 \%$ were passive smoker and most of the cases source was husband, $19.6 \%$ took smokeless tobacco or green tobacco, 55.8\% took tea and 5.8\% took coffee during pregnancy period. On logistic regression analysis passive smoking $(p=0.019)$ was independently associated with CHDs. Mother with passive smoking exposure had 1.5 times risk of developing CHDs compared to those who were not exposed. Conclusions: Passive smoking is significantly associated with CHDs.
\end{abstract}

Keywords: Passive smoking, Congenital Heart Defects, Bangladeshi population.

DOI: $10.51407 /$ mjpch.v27i1.120

\section{Introduction}

CHDs represent approximately one-third of all congenital anomalies and are the most common group of congenital malformations, affecting almost $1 \%$ of live births throughout the world and one of the leading causes of perinatal mortality $[1,2]$. Although advancement in paediatric cardiology and paediatric cardiac surgery have improved long term outcome and promised better quality of life, the etiology of most congenital heart defects is still unknown. It is difficult to establish the role of a single factor because the cause of a defect is believed to be multifactorial [3]. Clinicians and basic scientists
Received: 14 October 2020; Accepted revised manuscript: 26 April 2021

Published online: 29 June 2021

have long understood the sources of these cardiovascular developmental errors, and there is wide acceptance of the opinion that the etiology

\footnotetext{
'Division of Neonatal Cardiology, Department of Paediatric Cardiology, Bangladesh Institute of Child Health and Dhaka Shishu (Children) Hospital, Dhaka, Bangladesh.

${ }^{2}$ Bangladesh Institute of Child Health, Dhaka Shishu (Children) Hospital, Dhaka, Bangladesh.

${ }^{3}$ Department of Paediatric Cardiology, Dhaka Shishu (Children) Hospital, Dhaka, Bangladesh.

Corresponding Author:

Dr. Mohammad Abdullah Al Mamun, Division of Neonatal Cardiology, Department of Paediatric Cardiology,

Bangladesh Institute of Child Health and Dhaka Shishu (Children) Hospital, Sher-e-Bangla Nagar, Dhaka-1207, Bangladesh.

Tel.: +8801913475529 Email: mamundsh@gmail.com
} 
of CHD is complex and possibly lies within the interaction of environmental exposures and inherited factors [4]. Despite a strong heritable basis, a genetic etiology is identified in less than $20 \%$ of CHD cases [5]. However, lack of enough information about modifiable risk factors for malformations in fetal heart development has impeded the prevention of CHDs.

Recent epidemiologic studies have demonstrated an association between maternal lifestyle factors, specifically smoking, alcohol, use of drugs, body mass index, psychological factors and the risk of CHDs in offspring [6]. An exploration of the contribution of non-inherited risk factors that are potentially modifiable is particularly important in the context of the growing health burden of CHD [7].

Maternal smoking during pregnancy tended to increase the risk of congenital heart defects. A study by Alberman et al. was one of the first to report the association between maternal cigarette smoking and CHDs [8]. However, the evidence since then has been mixed, with some studies showing positive associations and others providing null results. Some meta-analyses have investigated the association between maternal smoking during pregnancy and CHDs in offspring. One meta-analysis published in 1999 found no association for all CHDs [9]. Hackshaw et al. estimated the effects of maternal smoking across a spectrum of birth defects including heart defects [10]. However, the study did not evaluate the effects of maternal smoking on CHD subtypes and dose-response relationships (i.e., increasing levels of smoking) were not assessed. A latest meta-analysis of studies published between 1971 and 2011 found positive association for all CHDs with maternal smoking [11].

Although several meta-analysis confirmed maternal active smoking was significantly associated with risk of fetal CHDs, association between maternal passive smoking and risk of CHDs is inconclusive. A meta-analysis of observational studies was conducted to assess the risk of CHDs associated with maternal active and passive smoking and paternal smoking and concluded that maternal active smoking, maternal passive smoking as well as paternal smoking all increased the risk of CHDs in offspring. All types of parental smoking were associated with the risk of congenital heart defects, with an increase of $74 \%$ for men smoking, $124 \%$ for passive smoking in women, and $25 \%$ for women smoking, compared to no smoking exposure [12].

Active smoking among mothers in Bangladesh is quite rare, while smoking prevalence among men is high. The Global Adult Tobacco Survey (GATS) founds that the prevalence of male who smoked are far greater than female in Bangladesh (among males $45.6 \%$ as compared to females $3.1 \%$ ). Use of smokeless tobacco products in Bangladesh was approximately the same among males (26.4\%) and females (27.9\%) [13]. Khan et al. [14] recently found large number of female tobacco user both smoker and chewer in their study.

Relatively less information has been reported on modifiable risk factors that may have an adverse effect on the cardiovascular development. This has made difficult to create population-based strategies to reduce the burden of illness from CHDs and for couples to choose lifestyles to reduce the risk of delivering a child with $\mathrm{CHDs}$. This study was therefore performed to investigate the effect of passive smoking on the risk of developing CHD in Bangladesh population.

\section{Materials and Methods}

This study was conducted from July 2018 to July 2019 in Dhaka Shishu (Children) Hospital. The study population was the parents of children who visited the outpatient department of Dhaka Shishu (Children) Hospital which is the largest tertiary hospital deals with patients referred from all over Bangladesh. Parents of the children with congenital heart disease visiting the out-patient department of Paediatric Cardiology Unit of Dhaka Shishu (Children) Hospital was considered as cases. Controls were taken from parents of the children who don't have congenital heart disease visited other out-patient departments of Dhaka Shishu (Children) Hospital. Exclusion criteria included children with associated syndromes or other major systemic diseases such as Down's syndrome, gastrointestinal and any severe abnormalities of the central nervous system; children with family history of CHD; and parents who refuse to get involved in the study or to comply with its requirement. Data were collected by using a structured questionnaire (Research Instrument) containing all the variables of 
interest. The questionnaire was finalized following pretesting. Along with sociodemographic factors, face-to-face interviews were conducted to collect information on maternal active and passive smoking along with coffee, tea and alcohol drinking. Maternal smoking was defined as reported smoking at least 1 cigarette per day [15]. Maternal smokeless or green tobacco use during pregnancy was also noted. Passive or second hand smoking was defined as maternal self-reported exposure to tobacco smoke at home, or workplace, or both, at least $15 \mathrm{~min} /$ day during the periconceptional period [16]. Coffee consumption was classified as 1 - 6 cup/week (10-100 mg/day) [17] and tea consumption was classified as 1 or more cup/day. To minimize recall bias of exposure by mothers, all cases and controls were recruited from the mothers whose child was $<1$ year old. Maternal coffee and tea consumption were examined as potential confounders and effect modifiers. Data was processed and analyzed using computer aided statistical software SPSS (Statistical Package for Social Sciences) Version 21. Chi square test $\left(X^{2}\right)$, Odds ratio (OR) and $95 \%$ confidence intervals (Cls) were calculated to estimate the associations between maternal passive smoking and all CHDs. Risk factors on bivariate analysis were introduced into a logistic regression model as independent factors and dependent variable was CHDs to find out the association between CHDs and maternal passive smoking.

\section{Results}

Total 260 children having CHD was enrolled as cases and 280 children having no heart disease was taken as control. Among children male were predominant in both groups. Most of the respondents were from rural area both in case (86.9\%) and control (80\%) group (Table 1).

Table 1. Sex and residence distribution of study population

\begin{tabular}{lccc}
\hline Baseline characteristics & & $\begin{array}{c}\text { Case } \\
(\mathbf{n = 2 6 0 )}\end{array}$ & $\begin{array}{c}\text { Control } \\
(\mathbf{n = 2 8 0 )}\end{array}$ \\
\hline Sex of children & Male & 144 & 178 \\
& & $(55.4)$ & $(63.6)$ \\
& Female & 116 & 102 \\
Residence & Rural & $(44.6)$ & $(36.4)$ \\
& \multirow{2}{*}{ Urban } & 226 & 224 \\
& & $(86.9)$ & $(80)$ \\
& & 34 & 56 \\
& & $(13.1)$ & $(20)$ \\
\hline
\end{tabular}

In case group 5.8\% took coffee and 55.8\% took tea during pregnancy period. No association was found between drinking coffee and tea during pregnancy period and CHD. No mother in this study was found to be alcoholic (Table 2).

Table 2. Association of consumption of coffee, tea and alcohol during pregnancy with CHDs

\begin{tabular}{lcccc}
\hline Maternal lifestyle factor & Case & $\begin{array}{c}\text { Control } \\
(\mathbf{n = 2 8 0 )}\end{array}$ & p value \\
\hline Coffee & Yes & $15(5.8)$ & $12(4.3)$ & 0.42 \\
& No & $245(94.2)$ & $268(95.7)$ & 0.60 \\
Tea & Yes & $145(55.8)$ & $150(53.6)$ & - \\
\multirow{3}{*}{ Alcohol } & No & $115(44.2)$ & $130(46.4)$ & \\
& Yes & 0 & 280 &
\end{tabular}

Chi square test $\left(X^{2}\right)$ was done to find out level of significance.

No mother in this study was found to be active smoker. In case group $51.9 \%$ were passive smoker and majority cases husband was the source of passive smoking, $19.6 \%$ took smokeless or green 
tobacco during pregnancy period. Maternal passive smoking $(p<0.05)$ [Table 3].

Table 3. Association of maternal smoking during pregnancy with CHD

\begin{tabular}{lllll}
\hline Smoking & & $\begin{array}{l}\text { Case } \\
(\mathbf{n = 2 6 0})\end{array}$ & $\begin{array}{l}\text { Control } \\
(\mathbf{n = 2 8 0})\end{array}$ & p value \\
\hline Maternal active smoking & Yes & 0 & 0 & - \\
& No & 260 & 280 & \\
Passive smoking & Yes & $135(51.9)$ & $120(42.9)$ & $0.03^{*}$ \\
(>15 min/day) & No & $125(48.1)$ & $160(57.1)$ & \\
Smokeless or green tobacco & Yes & $51(19.6)$ & $68(24.3)$ & 0.14 \\
& No & $109(80.4)$ & $212(75.7)$ & \\
\hline
\end{tabular}

Chi square test $\left(\mathrm{X}^{2}\right)$ was done to find out level of significance, ${ }^{*}$ Significant.

Risk factors on bivariate analysis were introduced into a logistic regression model as independent factors and dependent variable was CHDs. On logistic regression analysis passive smoking
( $p=0.019$ ) was independently associated with CHDs. Mother with passive smoking exposure had 1.5 times risk of developing CHDs compared to those who were not exposed (Table 4).

Table 4. Risk factors for developing CHDs using multivariate logistic regression

\begin{tabular}{llllll}
\hline Risk factors & B & SE & P value & OR & 95\% 'Cl' \\
\hline Sex of children & 0.350 & 0.178 & 0.05 & 0.705 & $0.497-0.999$ \\
$\begin{array}{l}\text { Maternal passive } \\
\text { smoking }\end{array}$ & 0.430 & 0.183 & 0.019 & 1.538 & $1.075-2.220$ \\
$\begin{array}{l}\text { Maternal use of } \\
\text { smokeless or green }\end{array}$ & -0.369 & 0.227 & 0.10 & 0.691 & $0.443-1080$ \\
$\begin{array}{l}\text { tobacco } \\
\text { Maternal coffee } \\
\text { consumption }\end{array}$ & 0.336 & 0.402 & 0.40 & 1.400 & $0.637-3.078$ \\
$\begin{array}{l}\text { Maternal tea } \\
\text { consumption }\end{array}$ & 0.340 & 0.196 & 0.082 & 1.405 & $0.957-2.061$ \\
\hline
\end{tabular}

\section{Discussion}

In this study $51.9 \%$ pregnant women were passive smoker, $19.6 \%$ took smokeless tobacco or green tobacco and 5.8\% took coffee during pregnancy period. None of the mother was smoker or alcoholic in this study. On logistic regression analysis passive smoking $(p=0.019)$ was independently associated with CHDs. Mother with passive smoking exposure had 1.5 times risk of developing CHDs compared to those who were not exposed.

Caffeine is a natural component of coffee, tea, cocoa and cola products. It is teratogenic in animal studies when administered at high concentrations. In human, caffeine and its metabolites easily cross the placenta and reach the fetus [18]. There is no evidence to support a teratogenic effect of caffeine in humans. In a systematic review investigating the association between maternal exposure to caffeine and risk of congenital anomalies, slight elevations were observed for associations between coffee intake and cardiovascular malformations, but not for the association between tea and cardiovascular malformations [17]. In this study no association was found between maternal intake of caffeine, tea, and the risk of developing CHDs in exposed infants.

Findings have shown that maternal smoking has adverse effects on the developing fetus, including hypoxia caused by carbon monoxide, nicotine and reduction in the supply of essential nutrients to the embryonic tissues [19]. Liu et al. [16] observed that the adjusted odds of exposure to 
environmental tobacco smoke (ETS) during pregnancy among all CHDs cases were $44 \%$ higher than non-cases. This findings are consistent with a prospective case-control study in Italy showing an increased CHD odds (odds ratio $2.1,95 \%$ confidence interval 1.3 to $3.5, \mathrm{p}$ 0.002 ) in children whose fathers were smokers ( $\geq 15$ cigarettes/day) [20]. Another case-control study conducted in Kaunas, Lithuania (1995-2005) showed that paternal smoking was associated with a $45 \%$ increased odds [21]. In this study CHD was significantly higher among infants who exposed to passive smoking and most of the cases father was the source of passive smoking. Passive smoking involves exposure to the same range of tobacco smoke toxins experienced by active smokers. Although at lower levels, it is likely that exposure to secondhand smoke also causes some or all of these complications but with lower levels of relative risk. Cigarette smoking contribute to the aetiology of CHD through induction of either male and female germ-cell mutation or interference with epigenetic pathways [22]. All forms of tobacco use and exposure, including chewing tobacco and secondhand smoke, significantly increased health hazard. Because the timing and mechanism of this effect is not clear, it is important to prevent passive smoking exposure in women before and during pregnancy [23].

Active smoking among mothers in Bangladesh is low, while smoking prevalence among men is high, but the use of smokeless tobacco products is approximately the same among males and females $[12,13]$. In this study large number of mothers took smokeless or green tobacco even in their pregnancy period. It was not found statistically significant in the development of CHDs. Evidence suggests that although smokeless tobacco are less hazardous than smoked tobacco, there is indeed reason to be cautious about the use of smokeless tobacco [24].

This study have some limitations. First, lack of adequate confounder adjustment. Few maternal lifestyle modification factors are studied here to rule out confounder. Controls from the same hospital was taken to minimize potential confounders. Second, there is lack of details on level of exposure and time of exposure of passive smoking along with level of consumption of coffee and tea. Future studies might need to consider measuring the level of exposure and timing of exposure. Third, the history of passive smoking exposure relied largely on the report by the parent of a child. There may be possibility of reporting bias in eliciting the history of passive smoking exposure. To minimize recall bias of exposure by mothers, all cases and controls were recruited from the mothers whose child was $<1$ year old. Finally it was a hospital based single center study.

Environmental tobacco smoke (ETS) is a substantial public health problem in Bangladesh. The adverse effects of ETS on CHDs and other health outcomes may be greatly reduced through intervention efforts, particularly to reduce paternal smoking prior to and during pregnancy [23].

\section{Conclusion}

Maternal passive smoking was shown to be significantly associated with an increased risk of CHD among exposed infants. Preventing passive smoking during peri-pregnancy is a priority for CHDs prevention.

\section{Funding}

This study was funded by Ministry of Science and Technology, Government of People's Republic of Bangladesh.

\section{References}

[1] Boneva RS, Botto LD, Moore CA, Yang Q, Correa A, et al. Mortality associated with congenital heart defects in the United States: Trends and racial disparities, 1979-1997. Circulation. 2001;103: 2376-81.

[2] Hoffman Jl, Kaplan S. The incidence of congenital heart disease. J Am Coll Cardiol. 2000;39: 1890-1900.

[3] Brent RL. Environmental causes of human congenital malformations: the pediatrician's role in dealing with these complex clinical problems caused by a multiplicity of environmental and genetic factors. Pediatrics. 2004;113: 957-68.

[4] van der Bom T, Zomer AC, Zwinderman $A H$, Meijboom FJ, Bouma BJ, Mulder BJ. The changing epidemiology of congenital heart disease. Nat Rev Cardiol 2011;8:50-60.

[5] Richards AA, Santos LJ, Nichols HA, Crider BP, Elder FF, Hauser NS, et al.Cryptic chromosomal abnormalities identified in 
children with congenital heart disease. Pediatr Res. 2008; 64:358-63.

[6] Feng Y, Yu D, Yang L, Da M, Wang Z, Lin Y, et al. Maternal lifestyle factors in pregnancy and congenital heart defects in offspring: Review of the current evidence. Italian Journal of Pediatrics. 2014; 40:85DOI: 10.1186/s13052014-0085-3.

[7] Jenkins KJ, Correa A, Feinstein JA, Botto L, Britt $A E$, Daniels $S R$, et al. Noninherited risk factors and congenital cardiovascular defects: current knowledge. Circulation. 2007;115:2995-3014.

[8] Alberman ED, Goldstein H. Possible teratogenic effect of cigarette smoking. Nature. 1971;231:529-30.

[9] Kallen K. Maternal smoking and congenital heart defects. Eur J Epidemiol. 1999;15:73137.

[10] Hackshaw A, Rodeck C, Boniface S. Maternal smoking in pregnancy and birth defects: $A$ systematic review based on 173687 malformed cases and 11.7 million controls. Hum Reprod Update. 2011;17:589-604.

[11] Lee LJ, Lupo PJ. Maternal smoking during pregnancy and the risk of congenital heart defects in offspring: A systematic review and metaanalysis. Pediatr Cardiol. 2013;34:398407.

[12] Zhao L, Chen L, Yang T, Wang L, WangT, Zhang $S$ et al. Parental Smoking and the Risk of Congenital Heart Defects in Offspring: An Updated Meta-Analysis of Observational Studies. Eur J Prev Cardiol 2019 Mar 23;2047487319831367. doi: $10.1177 / 2047487319831367$.

[13] Blecher E. The impact of tobacco advertising bans on consumption in developing countries. J Health Econ. 2008;27:930-42.

[14] Khan NR, Mahmood AR. Pattern of tobacco consumption and related factors among the people residing in a rural area. Bangladesh Med J. 2015; 44: 32-37.

[15] Flanders WD, Lally CA, Zhu BP, Henley $\mathrm{SJ}$, Thun MJ. Lung cancer mortality in relation to age, duration of smoking, and daily cigarette consumption: results from Cancer Prevention Study II Cancer Res. 2003;63:655662.
[16] Liu X, Nie Z, Chen J, Guo X, Ou Y, et al. Does maternal environmental tobacco smoke interact with social-demographics and environmental factors on congenital heart defects. Environmental Pollution 2018;234:214-22.

[17] Browne ML. Maternal exposure to caffeine and risk of congenital anomalies: A systematic review. Epidemiology. 2006;17:324-31.

[18] Grosso L, Bracken MB. Caffeine metabolism, genetics, and perinatal outcomes: A review of exposure assessment considerations during pregnancy. Ann Epidemiology.2005;15:46066.

[19] Andriani H, Kuo HW. Adverse effects of parental smoking during pregnancy in urban and rural areas. BMC Pregnancy Childbirth. 2014; 14:414.

[20] Cresci M, Foffa I, Ali LA, Pulignani S, Gianicolo EA, Botto N, et al. Maternal and paternal environmental risk factors, metabolizing GSTM1 and GSTT1 polymorphisms, and congenital heart disease. Am J Cardiol. 2011;108:1625-31.

[21] Kuciene R, Dulskiene V. Parental cigarette smoking and the risk of congenital heart septal defects. Med Kaunas Lith. 2010;46:635641.

[22] Leonardi-Bee J, Britton J, Venn A. Secondhand smoke and adverse fetal outcomes in nonsmoking pregnant women: A meta-analysis. Pediatrics. 2011;127:734-41.

[23] Gianicolo EAL, Cresci M, Ait-Ali L, Foffa I, Andreassi MG. Smoking and congenital heart disease: The epidemiological and biological link. Curr Pharm Des. 2010;16:2572-77.

[24] Bolinder G. Gilljam H. Smokeless Tobacco Health Hazards or Less Harm? In: Loddenkemper $\mathrm{R}$, Kreuter $\mathrm{M}$, editors. The Tobacco Epidemic, ed 2, rev. and ext. Prog Respir Res. Basel, Karger, 2015, vol 42, pp 243251. 